\title{
Análisis de la Deserción Estudiantil en el Programa Ingeniería de Alimentos de la Universidad de Cartagena durante el Periodo Académico 2009 - 2013
}

\author{
Diofanor Acevedo*, José D. Torres y Diego F. Tirado \\ Universidad de Cartagena, Facultad de Ingeniería, Programa de Ingeniería de Alimentos, Avenida el \\ Consulado, Calle 30 No. 48-152. Cartagena, Bolívar-Colombia (e-mail: diofanor3000@gmail.com) \\ * Autor a quien debe ser dirigida la correspondencia
}

Recibido Jul. 31, 2014; Aceptado Oct. 8, 2014; Versión final recibida Oct. 30, 2014

\begin{abstract}
Resumen
El objetivo de este trabajo fue analizar las causas de la deserción estudiantil en el programa de Ingeniería de Alimentos de la Universidad de Cartagena. Se usó información del Sistema de Prevención y Análisis de Deserción en Instituciones de Educación Superior, herramienta del Ministerio de educación de Colombia. Se incluyó también información obtenida mediante encuestas presenciales, por correo electrónico, y llamadas telefónicas a una muestra representativa de 60 estudiantes de edad promedio 22.1 años que abandonaron estudios. La deserción más alta se presentó en los primeros cuatro semestres. El promedio por semestres fue de $10.75 \%$, mientras que la acumulada de $55.87 \%$, lo cual estuvo por encima del promedio en Colombia para programas similares con $10.53 \%$ y $52.89 \%$ respectivamente. La mayoría de los estudiantes atribuyeron su decisión de interrumpir los estudios a su situación económica. Se requiere diseñar planes que posibiliten un acompañamiento integral de los estudiantes en riesgo para disminuir la deserción.
\end{abstract}

Palabras clave: deserción estudiantil, estudios universitarios, abandono de estudios, fracaso académico

\section{Analysis dropout of the student in the Program of Food Engineering at the University of Cartagena during the academic period $2009-2013$}

\begin{abstract}
The aim of this study was to analyze the causes of dropout in the Food Engineering Program at the University of Cartagena. Information from the System Analysis and Prevention Dropout in Higher Education Institutions, a tool of the Colombian Ministry of Education was obtained. Also, information gathered by classroom surveys, email questioning and telephone calls to a representative sample of 60 students of 22.1 years of age who had abandoned studies. The highest dropout occurred in the first four semesters. The average per semester was $10.75 \%$, while the cumulative $55.87 \%$, which was above the average for similar programs in Colombia with $10.53 \%$ and $52.89 \%$ respectively. Most students attributed the decision for discontinuing studies to their personal economic situation. This paper concludes that it is necessary to design plans that allow a more comprehensive support of students at risk for reducing dropout rates.
\end{abstract}

Keywords: student dropout, university studies, study abandon, academic failure 


\section{INTRODUCCIÓN}

La educación en general, y en especial la superior, aporta al ser humano elementos y posibilidades indispensables para su desarrollo personal, familiar y social; pero este propósito se ve afectado por fenómenos como la deserción académica (Bjerk, 2012), la cual se define como el hecho de que un número de estudiantes matriculados no siga la trayectoria normal en un determinado programa académico, lo que puede ser atribuido a diversas causas, tanto de tipo internas como externas de los mismos estudiantes (Díaz, 2008; Espinoza et al., 2012; Gibbs y Heaton, 2014). Sin embargo definir el concepto de deserción en forma puntual, presenta dificultades, debido a que no existen parámetros teóricos claros que lo delimiten, más allá del indicador con el que se refiere al ausentismo o abandono de un joven de la institución donde se matriculó para cursar el grado de escolaridad específico (Eicher et al., 2014).

El amplio espectro de actos que se pueden categorizar o rechazar dentro de los indicadores de deserción, representa una dificultad tanto para investigadores, como para hacedores de políticas educativas (Fernández-González, 2009; Fall y Roberts, 2012; De Witte et al., 2013). El problema se agrava, ya que la mayoría de las instituciones de educación superior, no poseen estudios sistemáticos que brinden información suficiente, oportuna y confiable en términos reales acerca de las causas que determinan que los estudiantes se retiren de la universidad antes de culminar satisfactoriamente su formación profesional (Korhonen et al., 2014). Para el sociólogo Vincent Tinto citado por Wietse et al., (2011) ninguna definición puede captar en su totalidad la complejidad de este fenómeno, quedando en manos de los investigadores la elección de la aproximación que se ajusta a sus objetivos y al problema por investigar.

Las altas tasas de deserción estudiantil universitaria, en los países occidentales contrastan fuertemente con los objetivos sociales y económicos que han sido formuladas por los funcionarios de los gobiernos y legisladores, con el fin de lograr un crecimiento económico sostenible (Bask y Aro, 2013; De Witte et al., 2013). En Colombia los índices de deserción producen una gran preocupación, pues no sólo la tasa de cobertura de la educación superior es baja, sino que de los pocos ya privilegiados que logran un cupo en la universidad sólo culminan su proceso, aproximadamente, el 50\% de ellos (Castaño et al., 2006; Gvirtz y Oría et al., 2010). Lo anterior significa que las tasas de egreso y titulación profesional del sistema educativo superior son relativamente bajas respecto de la proporción total de estudiantes que ingresan al medio (SPADIES, 2014).

Diversos autores afirman que la deserción tiene un origen multicausal y aunque los factores pueden ser comunes para este fenómeno, la magnitud e impacto de estos pueden variar según el contexto donde ocurran (Vera-Noriega et al., 2012; Lugo, 2013). Por ello garantizar el acceso a una formación profesional de calidad, no es sólo un asunto de ampliar cobertura; sino de desarrollar estrategias que permitan que quienes ingresen al sistema permanezcan en el, hasta culminar (De Witte y Rogge, 2013). Autores como Rinne y Järvinen, (2011), coinciden en aseverar que la deserción estudiantil ocasiona consecuencias, entre las que se destacan: en lo personal, la frustración y exclusión que sienten quienes se ven obligados, por una u otra causa, a abandonar su anhelo de convertirse en profesionales; en lo social, a generar más inequidad y desequilibrios; y en lo económico, los altos costos que ello acarrea (Wietse et al., 2011; Román, 2013).

Estudios sobre factores asociados a la deserción estudiantil, han encontrado que en la educación superior existen causas comunes con los demás niveles educativos, tales como los socioeconómicos, pérdida de sentido de la educación, dificultad para combinar trabajo y estudios, embarazos, conflictos con los docentes, falta de afecto, agresividad, entre otros. Además que las tasas más elevadas de deserción para la educación en Ingeniería por lo general se presentan en los primeros niveles (Castaño et al., 2006; Andrei et al., 2012; Bask y Aro, 2013; Guimarães et al., 2010; SPADIES, 2014).

En general, conociendo los factores asociados a esta problemática, será posible lograr un verdadero impacto en su disminución, dado que se podrán diseñar estrategias integrales que incluyan: acciones institucionales de revisión, ajuste a las políticas, prácticas pedagógicas, contenidos curriculares, mejorar la cualificación de los docentes, así como los asuntos de bienestar y convivencia universitaria, entre otros (Bjerk, 2012; Gibbs y Heaton, 2014). En el presente trabajo se utilizará el concepto de deserción estudiantil en relación con el abandono de un programa académico de educación superior por más de un año lectivo, a causas de factores internos y externos al ambiente educativo como problemas personales, familiares, económicos, y socioculturales. Teniendo en cuenta lo anterior el objetivo de este trabajo fue analizar el fenómeno de la deserción estudiantil en el programa Ingeniería de Alimentos de la Universidad de Cartagena, durante el periodo académico 2009-2013, para de esta manera poder esclarecer las principales causas que llevan a que los estudiantes abandonen sus estudios profesionales. 


\section{MATERIALES Y MÉTODOS}

\section{Población y Muestra}

Para el desarrollo de este estudio se empleó un diseño metodológico descriptivo, no experimental y de corte transversal, durante el periodo comprendido desde el primer semestre de 2009 hasta segundo semestre de 2013. La población objeto de estudio estuvo conformada por todos los estudiantes del Programa Ingeniería de Alimentos de la Universidad de Cartagena, que desertaron durante dicho periodo, la cual fue de 408. Para el cálculo del tamaño muestral, se utilizó la herramienta online, traducida y adaptada por González (2005), la cual cuenta con un nivel de confianza del 90\%, un margen de error del 10\%, una variabilidad positiva del $50 \%$ y variabilidad negativa del $50 \%$. Indicó un tamaño mínimo recomendado de 59 personas, sin embargo por efectos prácticos en este trabajo fueron seleccionados 60 estudiantes, distribuidos equitativamente, 30 hombres y 30 mujeres, quienes presentaron una edad promedio de 22,1 $\pm 0,39$ años.

\section{Instrumentos de la investigación}

Se utilizó información obtenida de reportes y documentos institucionales del SPADIES (Sistema para la prevención y análisis de la deserción en las instituciones de educación superior), herramienta del Ministerio de educación de Colombia. Al grupo de 60 estudiantes seleccionados ( $50 \%$ hombres y $50 \%$ mujeres) se les hicieron encuestas presenciales, por correo electrónico y llamadas telefónicas, dividiéndolos en grupos de 20 estudiantes para cada tipo de recolección. El instrumento utilizado fue un cuestionario compuesto por un total de 20 preguntas abiertas y flexibles distribuidas de la siguiente manera: 5 correspondiente a la dimensión personal, 5 haciendo referencia a la dimensión académica, 5 relacionadas con los factores socioeconómicos y 5 abarcando la dimensión institucional. De manera similar a lo realizado por Castaño et al., (2006) y Rodríguez y Hernández (2008). En el formato los estudiantes colocaron su nombre completo, semestre, identificación, dirección, teléfono y fecha de retiro.

\section{Análisis de datos}

Después de obtener la información se construyó una base de datos en una hoja de cálculo Excel 2013 para Windows, y se introdujeron las respuestas dadas por los estudiantes. Se llevó a cabo los procedimientos de depuración de los datos, necesarios para realizar los análisis correspondientes de porcentajes, media, desviación estándar.

\section{RESULTADOS Y ANÁLISIS}

El índice de deserción semestral permite establecer el comportamiento de este fenómeno con más detalle, en relación al número total de estudiantes desertores del programa de Ingeniería de Alimentos en el periodo t y el número total de estudiantes matriculados para el mismo periodo. Este se calculó de acuerdo a la Ecuación (1):

$\left(\frac{\mathrm{D}(\mathrm{t})}{\text { Ptotal }}\right) * 100$

Siendo $D_{t}$ los desertores en el periodo t y $P_{t}$ es la población total de estudiantes matriculados en el periodo t. La Figura 1 muestra el índice de deserción en el programa Ingeniería de Alimentos de la Universidad de Cartagena.

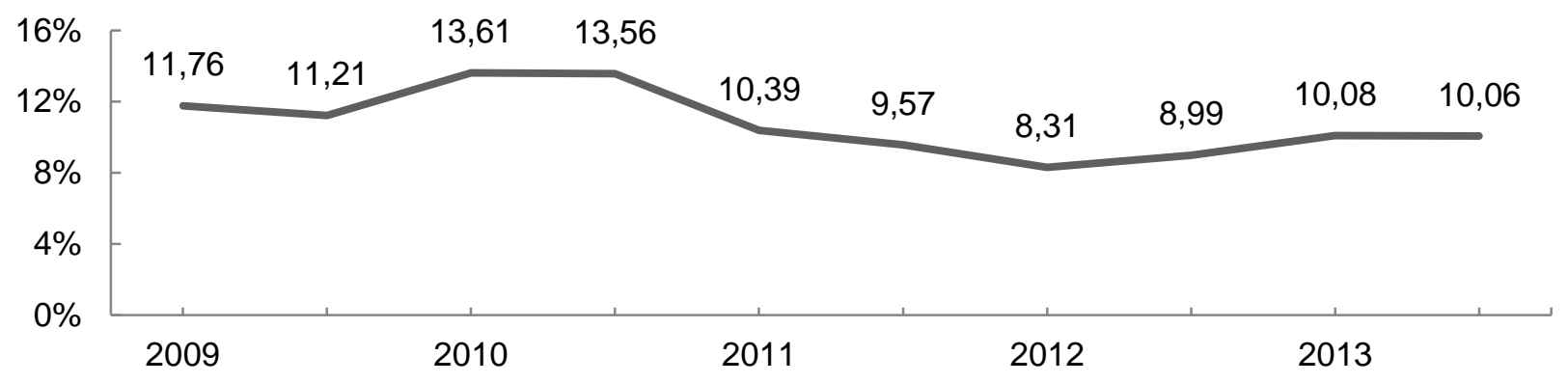

Fig.1: Índice de deserción en el programa Ingeniería de Alimentos de la Universidad de Cartagena

Se puede ver que el promedio de deserción por cohortes fue de $10,75 \%$, durante el periodo de estudio analizado. Si comparamos este dato con la deserción promedio nacional por cohortes para todos los 
programas de Ingeniería de Alimentos y afines, la cual es de 12,77\% según SPADIES (2014), podemos afirmar que está ligeramente debajo del porcentaje nacional. Sin embargo, si lo confrontamos con programas de Ingeniería de Alimentos acreditados en alta calidad, como la Universidad de Córdoba (7,48\%), Universidad de Antioquia (10,15\%), Universidad Tadeo Lozano $(9,87 \%)$, Universidad de Pamplona (10,65\%), Universidad de Caldas (10,24\%) y Universidad del Valle $(9,68 \%)$, observamos que la deserción al interior del programa se encuentra por encima, y a su vez es uno de los porcentajes promedios más altos a nivel institucional en la Facultad de Ingeniería de la Universidad de Cartagena, superado únicamente por el programa Ingeniería de Sistemas presencial $(15,50 \%)$, y en su orden se encuentran para el mismo periodo de estudio Ingeniería Civil (7,10\%) e Ingeniería Química (5,40\%) (SPADIES, 2014).

En la Figura 2 se observan los porcentajes de deserción en el programa Ingeniería de Alimentos respecto a los demás programas de la misma facultad y las Ingenierías en Colombia. Se puede notar que éste se encuentra cerca del promedio nacional, superado en gran medida para el último semestre por el programa Ingeniería de Sistema presencial con un máximo de (19,6\%) (SPADIES, 2014).

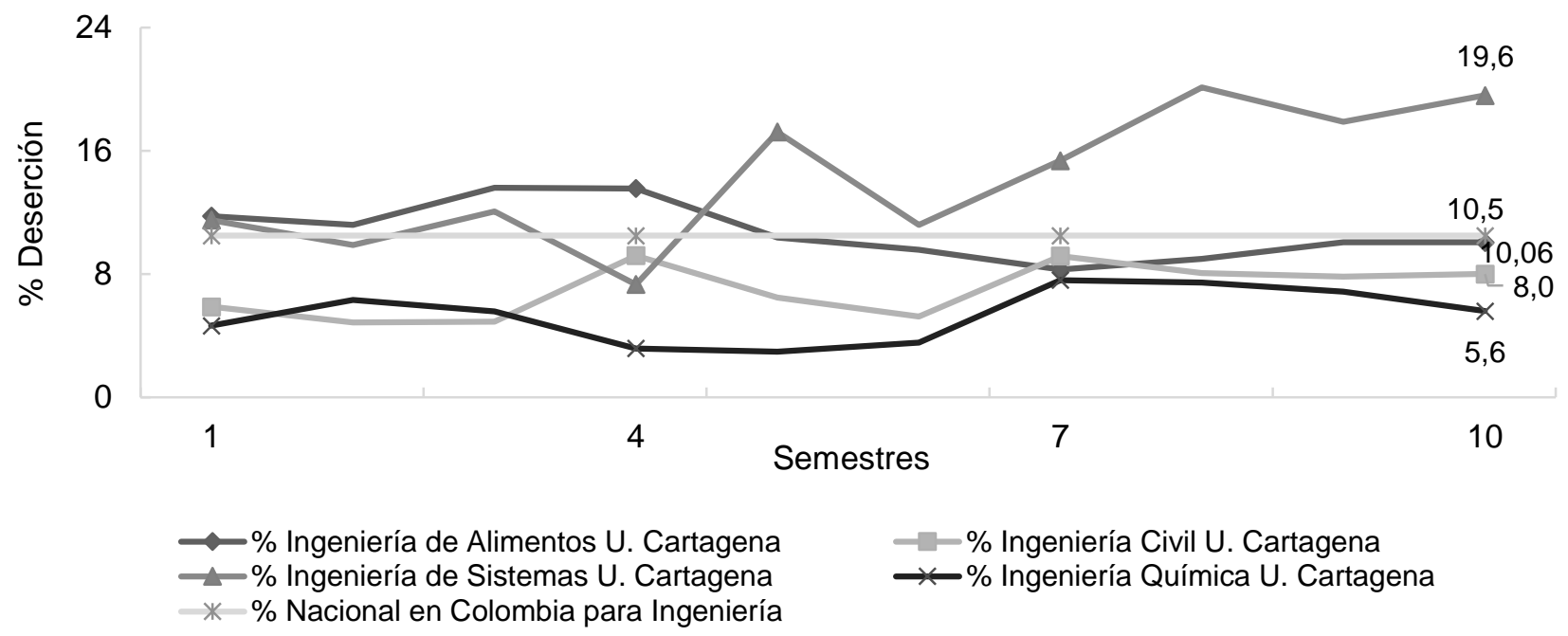

Fig. 2: Deserción en la facultad de Ingeniería de la Universidad de Cartagena

En la Figura 3 se compara el número de estudiantes egresados del programa de Ingeniería de Alimentos respecto al programa de Ingeniería Civil, para el mismo periodo académico de 2009 al 2013, ya que son los más antiguos de la facultad. Se puede observar una notable diferencia especialmente en los tres primeros semestres, y en el total de egresados, ya que Ingeniería de Alimentos tuvo 221 estudiantes, mientras que en Civil fueron 307, y con un promedio de 22 y 31 por semestre respectivamente.

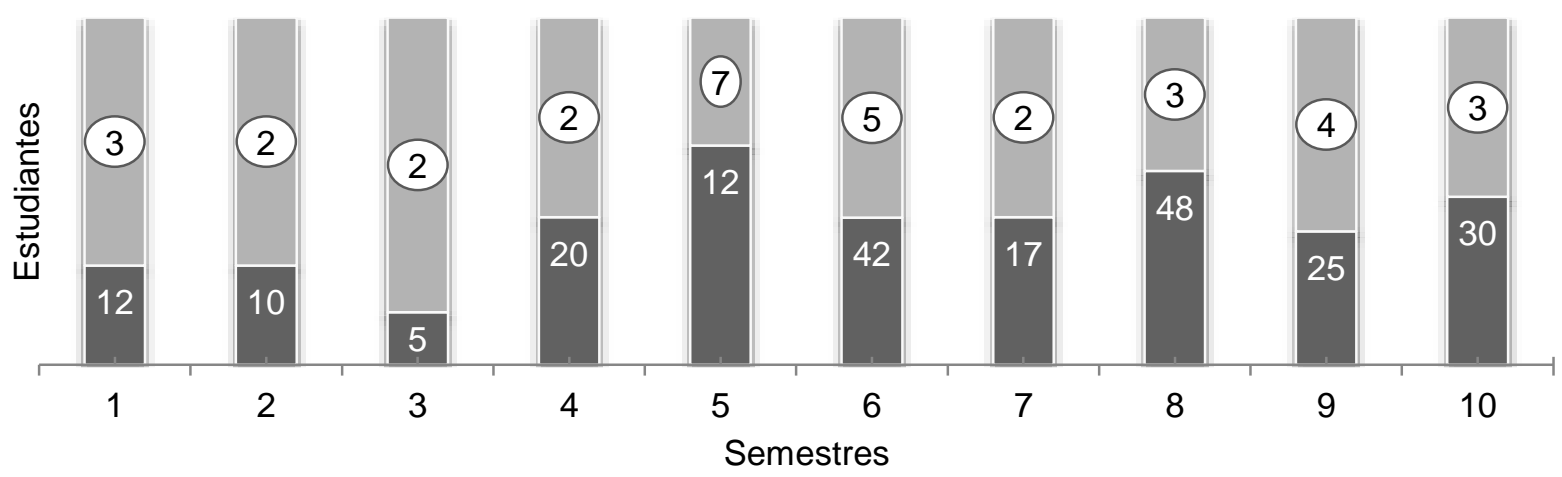

Fig. 3: Egresados en Ingeniería de Alimentos (color negro) en comparación con Ingeniería Civil (color gris) de la Univ. de Cartagena

En la Tabla 1 se analiza la deserción acumulada respecto a los estudiantes que ingresaron el primer semestre de 2009 y que de acuerdo a lo estipulado en el programa debieron obtener su título profesional en el segundo semestre de 2013 , debido a que se contabilizan exactamente diez semestres. Se puede notar que el programa sigue la tendencia en cuanto a la deserción que comúnmente se presenta en esta área de conocimiento en el país, llegando a un máximo de 55,87\% en décimo semestre, lo cual es más elevado que lo reportado en el SPADIES (2014) para carreras de Ingeniería de Alimentos, Agroindustrial y afines 
(52,89\%). Lo anterior es preocupante, pues significa que uno de cada dos estudiantes que empieza no culmina este tipo de Ingeniería. Para los demás programas de la facultad de Ingeniería de la Universidad de Cartagena, al consultar los datos en el SPADIES (2014) los porcentajes de deserción acumulada para Ingeniería de Sistema es el más elevado con un 62,93\%, casi igual al promedio nacional para Ingeniería de Sistemas, telecomunicaciones y afines (64,82\%), mientras que en Ingeniería Civil y Química son de 49,71 y $34,9 \%$ respectivamente, por lo que Ingeniería de Alimentos es el segundo más alto de la facultad en cuanto a la deserción estudiantil.

Tabla 1: Deserción acumulada en Ingeniería de Alimentos por semestres

\begin{tabular}{|l|c|c|c|c|c|c|c|c|c|c|}
\hline \multicolumn{1}{|c|}{ Semestre } & 1 & 2 & 3 & 4 & 5 & 6 & 7 & 8 & 9 & 10 \\
\hline $\begin{array}{l}\text { Ingeniería de Alimentos - } \\
\text { Universidad de Cartagena }\end{array}$ & 11,76 & 19,33 & 24,41 & 35,34 & 40,56 & 42,78 & 45,86 & 49,09 & 52,42 & 55,87 \\
\hline $\begin{array}{l}\text { Ingeniería de Alimentos afines, } \\
\text { promedio en Colombia }\end{array}$ & 22,04 & 27,04 & 34,04 & 42,04 & 44,21 & 46,21 & 47,21 & 48,21 & 49,21 & 52,89 \\
\hline
\end{tabular}

Según SPADIES (2014) en estas áreas del conocimiento Ingeniería y afines, especialmente durante los cuatro primeros semestres, se concentra la mayor deserción de la educación superior en Colombia, y a su vez afirma que uno de cada cinco estudiantes se retira en primer semestre o emigra hacia otras áreas. Lo que coincide con lo descrito por Cortes et al., (2011), quienes realizaron un estudio hecho en los nueve programas de la facultad de Ingeniería de la Universidad Nacional de Colombia entre los años 2002 y 2006. Estos autores encontraron que el $72,35 \%$ de los estudiantes que se retiraron lo hicieron en los primeros cuatro semestres. Por ello indicaron que es en esta etapa donde se debe hacer mayor énfasis en el acompañamiento académico. Por su parte Silva-Laya (2011), quien hizo una revisión extensa en la que analiza la importancia del primer año universitario como un tramo crítico en la definición de trayectorias estudiantiles exitosas, señaló que el primer año constituye un punto nodal en la decisión de abandonar o proseguir los estudios y, en ello, el involucramiento o compromiso del joven con sus estudios es determinante, ya que los jóvenes enfrentan dificultades que responden a factores externos a los programas académicos, pero también a variables propias como la autoestima. Y en las consideraciones finales afirma que la mejor estrategia para disminuir la deserción es brindar una atención integral y una educación de buena calidad.

Las Figuras 4 y 5 muestran la distribución porcentual de los factores de abandono académico para los estudiantes consultados. Se puede ver el predominio de los factores económicos, especialmente en los hombres, donde se estuvo un 5,7\% por encima a lo reportado para las mujeres. Es importante resaltar que de las mujeres consultadas el $22,3 \%$ indicaron que no le gustó la Ingeniería de Alimentos como profesión y la forma como se impartía en la Universidad de Cartagena, mientras que el porcentaje en los hombres fue del $13,3 \%$. Estos estudiantes consideraron que el programa no iba en sintonía con sus intereses y habilidades profesionales, además el pensum les parecía obsoleto y rígido, y mencionaron que la metodología de enseñanza fomentaba la baja calidad educativa, por tales motivos decidieron desertar. Estos resultados pueden ser atribuibles al desconocimiento de la carrera, y a una deficiente orientación vocacional al momento de escoger la profesión. Lo que coincide con lo reportado por Ensminger y Slusarcick (2002), quienes formulan que la orientación profesional y vocacional implica el análisis de las habilidades, intereses y su coherencia en un campo de formación. Es decir, se espera que la carrera elegida sea del interés del estudiante, pero no basta con la motivación hacia el contenido, si no que se requiere que la persona tenga habilidades para la profesión.

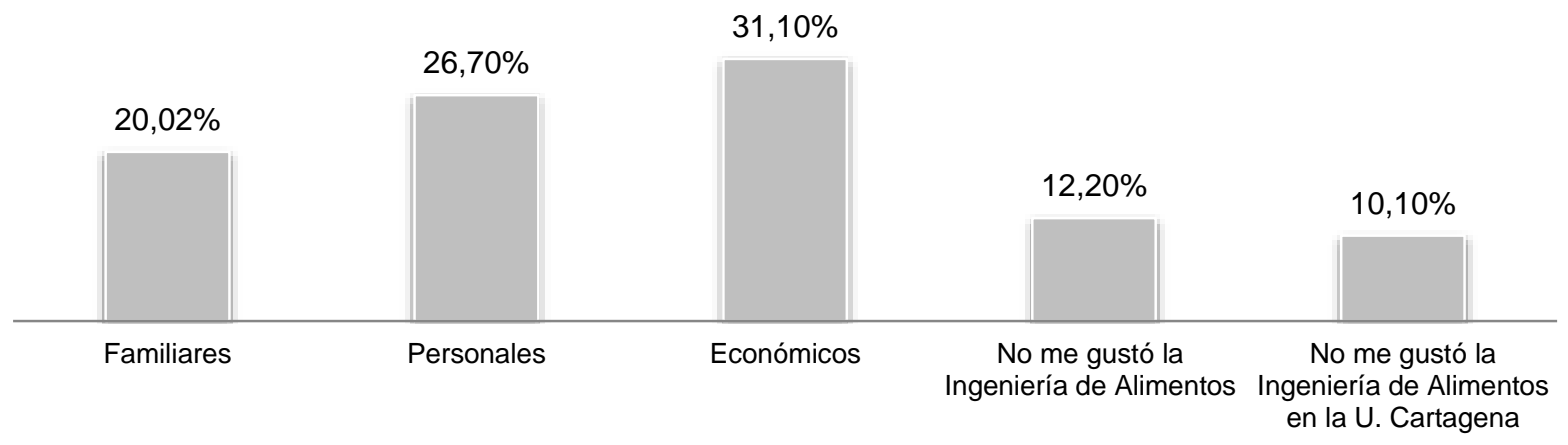

Fig. 4: Distribución porcentual factores de abandono académico para mujeres 


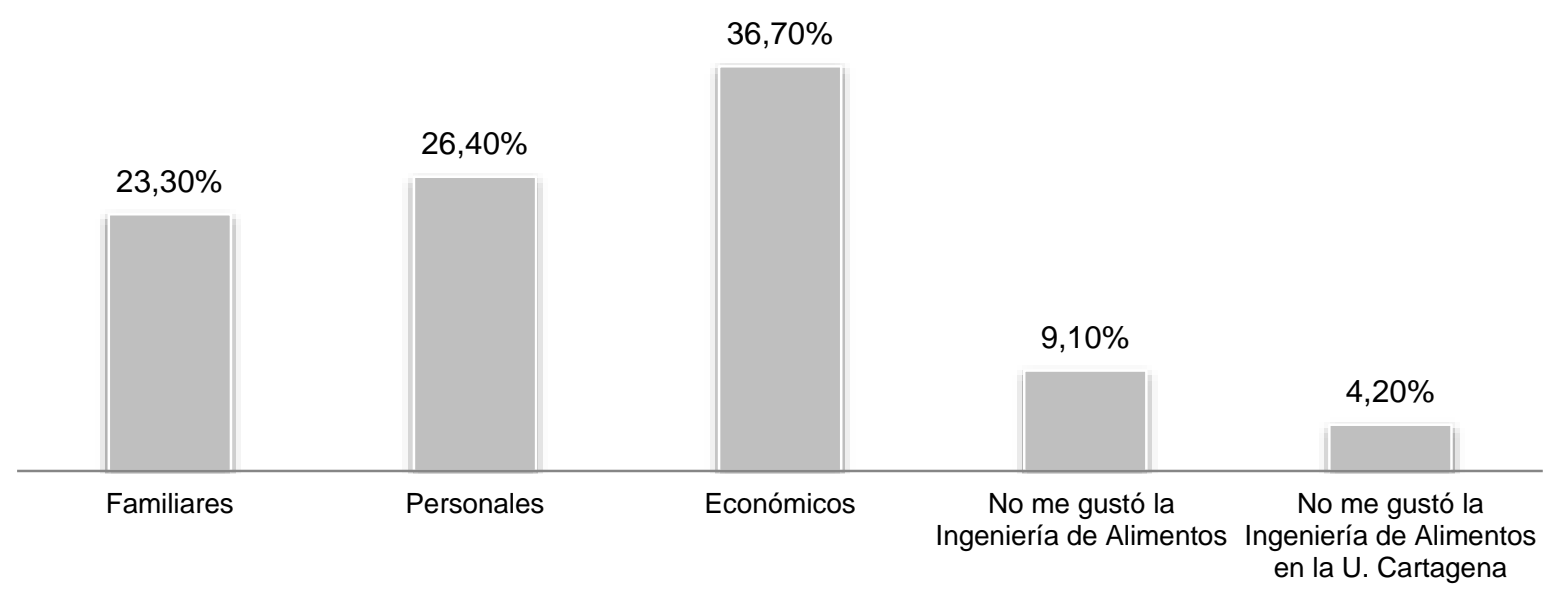

Fig. 5: Distribución porcentual factores de abandono académico para hombres

De esta manera, la coherencia entre las habilidades y los intereses está estrechamente vinculada con la permanencia del estudiante en su formación académica. Cuando falla la habilidad o el interés, generalmente hay dificultades en la permanencia de un estudiante en la carrera que había elegido. Teniendo este modelo teórico como referente, toma más sentido que los estudiantes que desertaron en el programa Ingeniería de Alimentos, consideren que no tenían las habilidades académicas exigidas por la carrera. Para la muestra de estudiantes consultadas, no se observaron diferencias substanciales entre hombres y mujeres, en los factores de tipo familiar y personales.

Estos resultados coinciden con los hallazgos de Castaño et al., (2006), quienes afirman que existen dimensiones explicativas del fenómeno de la deserción estrechamente vinculadas con las condiciones económicas de las familias colombianas, en tanto que las características de la población señalan bajos ingresos económicos familiares, hogares monoparentales o problemas de inserción laboral. Así mismo, la investigación de Castro et al., (2006) llevada a cabo con estudiantes chilenos, mostró desde el enfoque de la injusticia social, que los jóvenes entran al sistema educativo con ciertas condiciones iniciales asociadas al nivel de ingresos económicos. Para estos investigadores, la pobreza se configura en un factor de riesgo para la deserción universitaria, puesto que el problema no se restringe al acceso a fuentes de información, sino a que muchos de los estudiantes con bajos ingresos económicos se ven abocados a trabajar para poder asistir a las clases con los recursos mínimos que exige su formación profesional. En línea con estos resultados, las investigaciones realizadas por Espinoza et al., (2012), Román (2013) y Rodríguez y Hernández (2008) encontraron que los estudiantes que trabajan y provienen de familias de bajos recursos económicos tienen mayores riesgos de deserción. Las investigaciones concluyen que es preocupante el acceso y permanencia de los más pobres en la educación superior y la necesidad de trabajar influye negativamente sobre la permanencia del estudiante en las instituciones.

Del total de encuestados, el 55\% indicaron provenir de colegios no oficiales. De estos, el $57 \%$ eran mujeres, mientras que en los hombres este porcentaje fue del 53\%. Por lo tanto el lugar de procedencia en lo que respecta a la naturaleza de la institución educativa influye en la deserción, al interior del programa Ingeniería de Alimentos, lo que coincide con Castaño et al., (2006) en su investigación sobre la deserción en la facultad de Ingeniería de la Universidad Antioquia (Colombia), este afirma que los estudiantes jóvenes, hombres, y solteros o aquellos que han sufrido alguna calamidad familiar, tienen un mayor riesgo de desertar. $Y$ en términos académicos, la falta de orientación, el haber egresado de un colegio privado para estudiar en una universidad pública, el tener un bajo rendimiento académico, el no tener experiencia en la educación superior o el haber desertado de otra universidad, incrementan el riesgo de deserción. Estos resultados también coinciden con lo reportado por Canales y De los Ríos (2007), en su estudio sobre los factores explicativos de la deserción universitaria en Chile. Por su parte Silva (2005), plantea que el fenómeno de la deserción universitaria no sólo está en relación con los factores económicos o académicos, sino con el compromiso de los profesores en la formación de los estudiantes; lo cual constituye un claro referente de la gestión en el logro del mejoramiento del compromiso docente.

Mediante la entrevista telefónica se logró contactar a los desertores, con el fin de indagar acerca de su condición actual. En la Figura 6 se observa que un $81,67 \%$ de los desertores se dedicó a estudiar. En general solamente el $20,83 \%$ continuó con su vocación y su expectativa de culminar la Ingeniería de Alimentos, ya sea en la Universidad de Cartagena u otra institución. Este panorama aclara las causas de la deserción, y se comprueba el hecho de que muchos indicaron que el programa no llenaba sus expectativas 
y decidieron dedicarse a estudiar otra carrera $(43,50 \%)$. A su vez otros resolvieron intentarlo nuevamente, reingresando al programa (16,67\%), ya que, a pesar de los problemas de rendimiento académico, consideraron que esto era lo deseaban para sus vidas profesionales, lo que se comprueba al establecer un comparativo de sus situaciones académicas antes y después del reingreso, del cual se observó que muchos mejoraron considerablemente su rendimiento, pues pasaron de ser deficientes a ser muy buenos, en términos de promedio académico acumulado.

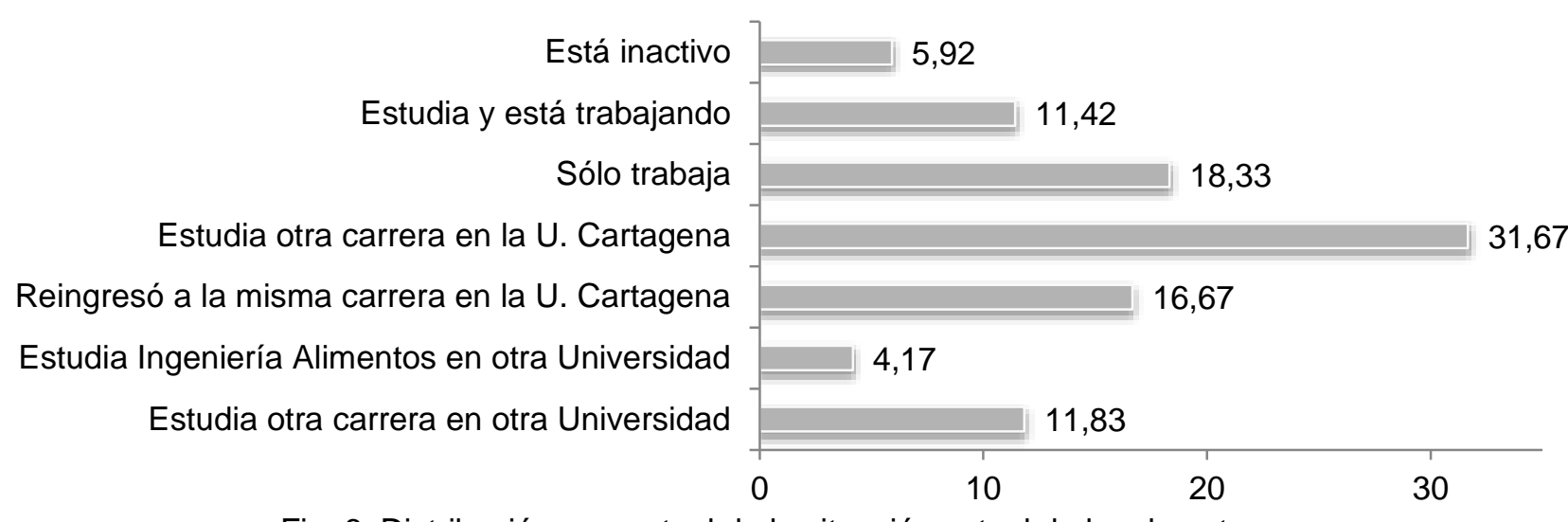

Fig. 6: Distribución porcentual de la situación actual de los desertores

\section{CONCLUSIONES}

En el programa Ingeniería de Alimentos de la Universidad de Cartagena, se presenta el fenómeno de la deserción, especialmente durante los primeros semestres; en lo cual influye las condiciones económicas de los estudiantes, problemática que en este estudio se hizo evidente, en un $31,10 \%$ para las mujeres y un $36,7 \%$ en hombres. Esto se encuentra en concordancia con otros estudios realizados en Colombia y en Latinoamérica. Por ello es importante la realización e implementación de estrategias como: tutorías académicas, acompañamiento psicológico y ayudas económicas a los estudiantes en riesgo, que posibilite mayor retención al interior del programa, así como mejores tasas de titulación profesional en esta Ingeniería. Teniendo en cuenta los factores de deserción presentados al interior del programa Ingeniería de Alimentos esto servirá para realizar un software que permita prevenir la deserción de manera temprana.

\section{REFERENCIAS}

Andrei, T., D. Teodorescu y B. Oancea, Quantitative methods used to identify the causes of school dropout in EU countries, Procedia - Social and Behavioral Sciences, 31(2), 188-192 (2012).

Bask, A. y S. Aro, Burned out to drop out: exploring the relationship between school burnout and school dropout, European Journal of Psychology of Education, 28(1), 511-528 (2013).

Bjerk, D., Re-examining the impact of dropping out on criminal and labor outcomes in early adulthood. Economics of Education Review, 31(1), 110-122 (2012).

Canales, A. y D. De los Ríos, Factores explicativos de la deserción universitaria. Revista Calidad en la Educación, Ministerio de Educación de Chile, 26 (1), 173-201 (2007).

Castaño, E., S. Gallón y J. Vázquez, Análisis de los factores asociados a la deserción estudiantil en la educación superior: un estudio de caso, Revista de Educación, 345, 255-280 (2006).

Castro, R. y G. Rivas, Estudio sobre el fenómeno de la deserción y retención escolar en localidades de alto riesgo, Sociedad Hoy, 011, 35-72 (2006).

Cortés, H., L. Gallego y G. Rodríguez, La Facultad de Ingeniería hoy: una aproximación hacia la construcción de indicadores académicos, Ingeniería e Investigación, 31(1), 74-90 (2011).

De Witte, K. y otros cuatro autores, A critical review of the literature on school dropout, Educational Research Review, 10 (1), 13-28 (2013). 
De Witte, K. y N. Rogge, Dropout from secondary education: All's well that begins well, European Journal of Education, 47(4), 1-20 (2013).

Díaz, Ch., Modelo conceptual para la deserción estudiantil universitaria chilena. Revista Estudios Pedagógicos, Universidad Austral de Chile, 34(2) (2008).

Eicher, V., C. Staerklé y A. Clémence, I want to quit education: A longitudinal study of stress and optimism as predictors of school dropout intention, Journal of Adolescence, 37(7), 1021-1030 (2014).

Ensminger, M. y A. Slusarcick, Paths to high school graduation or dropout: a longitudinal study, Sociology of Education, 65, 95-113 (2002).

Espinoza, O. y otros tres autores, Factores familiares asociados a la deserción estudiantil en Chile, Revista de Ciencias Sociales, 18(1), 136-150 (2012).

Fall, A. y G. Roberts, High school dropouts: Interactions between social context, self-perceptions, school engagement, and student dropout, Journal of Adolescence, 35(1), 787-798 (2012).

Gibbs, B. y T. Heaton, Dropout from primary to secondary school in Mexico: A life course perspective, International Journal of Educational Development, 36(1), 63-71 (2014).

González, M., Calculadora del tamaño de la muestra, Traducida y adaptada () 2004 by Raosoft, Inc. http://www.mey.cl/html/samplesize.html. Acceso: 20 de Julio (2006).

Guimarães, J. y B. Sampaion, What is behind University Dropout Decision in Brazil? A Bivariate Probability Model, The Empirical Economics Letters, 9(1), 601-608 (2010).

Gvirtz, S. y A. Oría, Evaluar el rendimiento interno y académico: un desafío para la macro y el micro político, lecciones a partir de un estudio de caso. Revista Iberoamericana de Evaluación Educativa, 3(2), 127-144 (2010).

Korhonen J., K. Linnanmäki y P. Aunio, Learning difficulties, academic well-being and educational dropout: A person-centred approach, Learning and Individual Differences, 31(2), 1-10 (2014).

Lugo, B., La deserción estudiantil: ¿realmente es un problema social? Revista de Postgrado FACE-UC, 7(12), 289-309 (2013).

Rinne, R. y T. Järvinen, Dropout and completion in upper secondary education in Finland, School dropout and completion, International comparative studies in theory and policy, 1(2), 215-232 (2011).

Rodríguez, J. y M. Hernández, La deserción escolar universitaria en México. La experiencia de la universidad autónoma metropolitana, Revista Electrónica Actualidades Investigativas en Educación, 8(001), 11-31 (2008).

Román, M.C., Factores asociados al abandono y la deserción estudiantil en américa latina: una mirada en Conjunto, Revista Iberoamericana sobre Calidad, Eficacia y Cambio en Educación, 11(2), 33-59 (2013).

Silva, R., Deserción: ¿competitividad o gestión? Revista Lasallista de Investigación, 2(002), 64-69 (2005).

Silva-Laya, M., El primer año universitario: Un tramo crítico para el éxito académico, Perfiles educativos, 33, 102-114 (2011).

SPADIES, Sistema para la Prevención de la Deserción en las Instituciones de Educación Superior en Colombia, (2014). Http: //spadies.mineducacion.gov.co/spadies/consultas.html. Acceso: 22 de julio (2014).

Vera-Noriega, J. y otros cinco autores, Factores asociados al rezago en estudiantes de una institución de educación superior en México, RIES, 3(7), 41-56 (2012).

Wietse, V. y otros tres autores, ¿Desertores o decepcionados? Distintas causas para abandonar los estudios universitarios, Revista de la educación superior, 40(4), 160-166 (2011). 